\title{
Discovery of Potent, Reversible MetAP2 Inhibitors via Fragment Based Drug Discovery and Structure Based Drug Design - Part 2
}

\author{
$\underline{\text { Christopher McBride }}^{\mathrm{a}}$, Zacharia Cheruvallath ${ }^{\mathrm{a}}$, Mallareddy Komandla ${ }^{\mathrm{a}}$, Mingnam Tang ${ }^{\mathrm{a}}$, Pamela \\ Farrell $^{\mathrm{b}}$, J. David Lawson ${ }^{\mathrm{c}}$, Darin Vanderpool ${ }^{\mathrm{b}}$, Yiqin Wu ${ }^{\mathrm{b}}$, Douglas R. Dougan ${ }^{\mathrm{d}}$, Artur \\ Plonowski $^{\mathrm{b}}$, Corine Holub ${ }^{\mathrm{b}}$, and Chris Larson ${ }^{\mathrm{b}}$ \\ ${ }^{a}$ Medicinal Chemistry, ${ }^{b}$ Biological Sciences, ${ }^{c}$ Computational Sciences, ${ }^{d}$ Structural Biology, Takeda \\ California
}

\begin{abstract}
Methionine aminopeptidase-2 (MetAP2) is an enzyme that cleaves an N-terminal methionine residue from a number of newly synthesized proteins. This step is required before they will fold or function correctly. Pre-clinical and clinical studies with a MetAP2 inhibitor suggest that they could be used as a novel treatment for obesity. Herein we describe the discovery of a series of pyrazolo[4,3b]indoles as reversible MetAP2 inhibitors. A fragment-based drug discovery (FBDD) approach was used, beginning with the screening of fragment libraries to generate hits with high ligand-efficiency (LE). An indazole core was selected for further elaboration, guided by structural information. SAR from the indazole series led to the design of a pyrazolo[4,3-b]indole core and accelerated knowledge-based fragment growth resulted in potent and efficient MetAP2 inhibitors, which have shown robust and sustainable body weight loss in DIO mice when dosed orally.
\end{abstract}

In our previous communication ${ }^{1}$, we described the rationale for our interest in MetAP2 as a target to treat obesity and disclosed an indazole fragment hit with moderate affinity (1, MetAP2 $\mathrm{pIC}_{50}$ of 5.6), and a high LE and lipophilic ligand efficiency (LLE) due to its small size and low lipophilicity. In that communication we identified interactions vital to potency, most importantly those at 6-position of the indazole core, which can generate gains in affinity of $>100$ fold over the unsubstituted core (Fig. 1, compound 2 vs. 3 ).

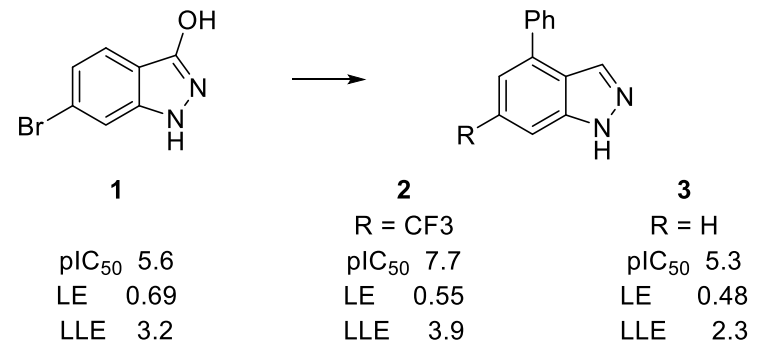

Fig. 1 Indazole series elaboration

In this paper, we disclose the structure based design of a new scaffold as part of our efforts in identifying multiple chemotypes for our program. This led to the identification of several potent pyrazolo[4,3b]indoles, as selective, orally bioavailable, and reversible MetAP2 inhibitors.

The co-crystal structure of an indazole ${ }^{1}$ bound to MetAP2 showed the N2-nitrogen of the indazole ring coordinates with one of the active site metal ions while the N1-nitrogen makes a water mediated interaction with the other active site metal. The 6-position substituent on the indazole ring is oriented to fill the adjacent hydrophobic site that is typically filled by the terminal methionine of the endogenous ligands for MetAP2. Filling this pocket yields the aforementioned potency gains of up to 100-fold. Installing an aryl group at the 4 position of the indazole core fills a hydrophobic cleft between Tyr444 and His-339 and boosts potency (5-10 fold).

In parallel to our work on the indazoles, we sought a novel chemical scaffold and designed a pyrazolo[4,3b]indole core which we hypothesized could satisfy all of the key interactions seen in the indazole series 
(Fig. 2, indazole core overlaid with model of pyrazolo[4,3-b]indole core). By utilizing the same pyrazole warhead we expected to maintain the interactions with the metal ions in the active site of MetAP2. The size of the tricyclic core would cause the phenyl ring to protrude even deeper into the lipophilic pocket, though still leaving room for small substitutions around the ring (7-fluorinated example shown).

Additionally, this new scaffold offered the opportunity for substitution at N4, which would point in nearly the same vector as the indazole 4-substitutions.

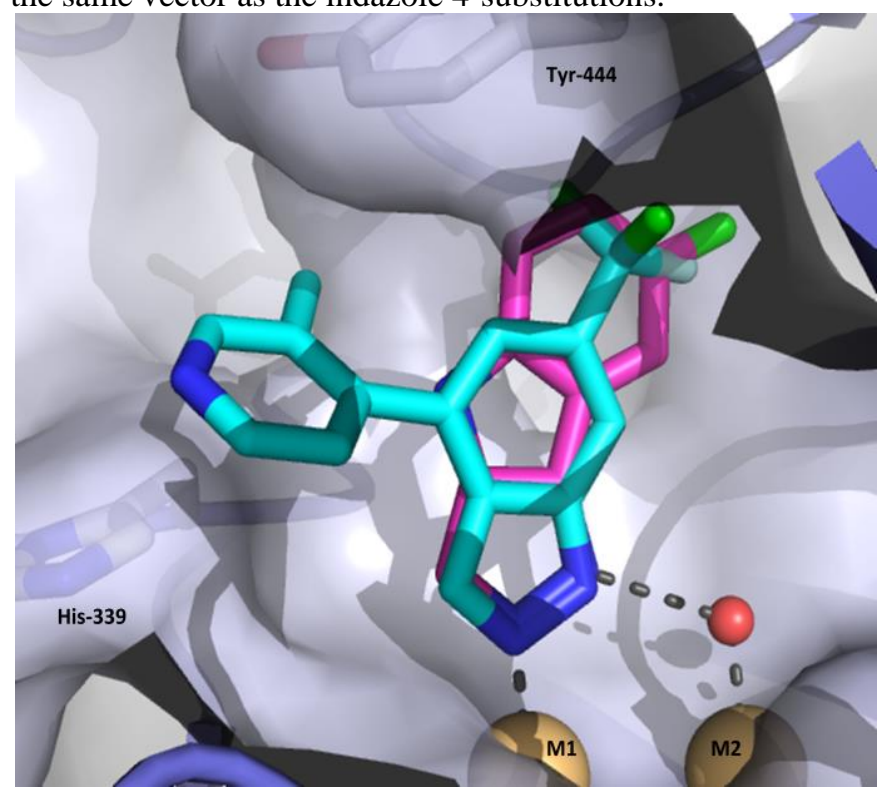

Fig. 2 Overlay of pyrazolo[4,3-b]indole model with indazole co-crystallized with MetAP2 (pdb code: 5JI6)

Previous work by Pudlo et.al. utilized a thermally decomposed azide for nitrene insertion and ring formation of fused aromatic indole heterocycles ${ }^{2}$. We utilized a similar strategy to access our target molecules. A representative synthesis for this series is described in Scheme 1. Starting with the diazotization of a substituted 2-bromoaniline (A) followed by treatment with sodium azide affords a substituted 1-azido-2-bromobenzene (B). This intermediate was then cross-coupled with N1-THP protected pyrazole-5-boronic ester and then thermally cyclized to the substituted N1-THP protected pyrazolo[4,3-b]indole (D). The N4-aryl substitution was installed using copper thiophene carboxylate to catalyze an Ullmann reaction, which was followed by removal of the THP group with methanolic $\mathrm{HCl}$ to afford the desired product $(\mathbf{F}){ }^{3}$<smiles>Nc1cc[R]cc1Br</smiles>

A
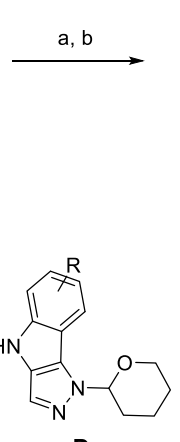

D

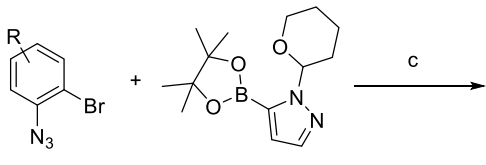

B

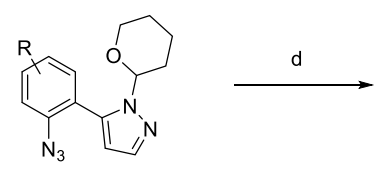

C

Scheme 1. Synthesis of 4-substituted pyrazolo[4,3-b]indoles: a) $\left.\mathrm{NaNO}_{2}, \mathrm{HCl}, \mathrm{b}\right) \mathrm{NaN}_{3}$ $70-88 \%$ yield over 2 steps, c) $\mathrm{Pd}\left(\mathrm{PPh}_{3}\right)_{4}, \mathrm{Na}_{2} \mathrm{CO}_{3}$, dioxane, heat $60 \%$ yield, d) dichlorobenzene, $170^{\circ} \mathrm{C}, 2 \mathrm{~h}$ $30 \%$ yield, e) $\mathrm{CuTC}, \mathrm{Cs}_{2} \mathrm{CO}_{3}, \mathrm{DMF}, 180^{\circ} \mathrm{C}, 2$ days, f) $\mathrm{MeOH}, \mathrm{HCl}, 10-70 \%$ yield over 2 steps 
Screening $^{3}$ of the unsubstituted pyrazolo[4,3-b]indole (5) showed that it had lower potency, though owing to the lower MW and lipophilicity, still displayed LE and LLE which would make this an attractive starting point (4, Fig. 3). As shown in Fig. 2, modeling ${ }^{4}$ also suggested that there was room for small substitutions at the 6 and 7-positions of the tricyclic core. We were encouraged that when a fluorine atom was installed at the 7-position of the tricyclic scaffold (6) we measured a greater than 10 fold increase in potency and saw a corresponding boost in efficiency.<smiles>FC(F)(F)c1ccc2cn[nH]c2c1</smiles>

4

$\mathrm{pIC}_{50} 7.3$

LE $\quad 0.77$

LLE $\quad 5.1$<smiles>c1ccc2c(c1)[nH]c1cn[nH]c12</smiles>

5 $\mathrm{plC}_{50} \quad 6.0$ LE $\quad 0.68$ LLE $\quad 4.6$<smiles>Fc1ccc2[nH]c3cn[nH]c3c2c1</smiles>

6

$\mathrm{pIC}_{50} 7.1$

LE $\quad 0.74$

LLE $\quad 5.5$

Fig. 3 Progression from indazole fragment to pyrazolo[4,3-b]indole core.

To explore the SAR at the 6 and 7 positions, we prepared a small set of $4 \mathrm{~N}$-arylated cores with different halogen substitutions and tested them in our enzymatic and cellular assays ${ }^{3}$. The HUVEC assay used is not a proliferation assay, but rather verifies target engagement by measurement of the accumulation of N-Met 14-3-3 $\gamma$ which is a client protein of MetAP2. While the fluoro and chloro substitutions tested showed similar activity against MetAP2, the 7-fluoro analog (7) showed superior cellular activity so this substitution was selected for use going forward (Table 1). Additionally, these relatively small inhibitors do display potent inhibition of CYP1A2.

Table 1. SAR of 6 and 7 position halogenations

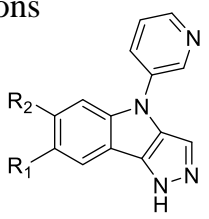

\begin{tabular}{ccccccc}
\hline Compd. & Structure $\left(\mathrm{R}^{1}, \mathrm{R}^{2}\right)$ & $\mathrm{clog} \mathrm{D}$ & $\begin{array}{c}\mathrm{MetAP2} \\
\mathrm{pIC}_{50}\end{array}$ & $\mathrm{LE}$ & $\mathrm{pEC}_{50}$ & $\begin{array}{c}\mathrm{CYP} 1 \mathrm{~A} 2 \\
\mathrm{pIC}_{50}\end{array}$ \\
\hline $\mathbf{7}$ & $\mathrm{F}, \mathrm{H}$ & 1.2 & 7.9 & 0.57 & 7.0 & 6.9 \\
$\mathbf{8}$ & $\mathrm{F}, \mathrm{F}$ & 1.1 & 8.1 & 0.55 & 5.9 & 7.3 \\
$\mathbf{9}$ & $\mathrm{H}, \mathrm{Cl}$ & 1.7 & 8.3 & 0.59 & 5.0 & 7.2 \\
\hline
\end{tabular}

From our previous work on the indazole series of MetAP2 inhibitors, we had a wealth of data (SAR, in vitro safety, microsomal stability, etc.) which has been discussed in the first part of this publication series (we found that aryl substitutions with ortho substituents were the most potent and stable in human liver microsomes). As the vector from the 4 position of both cores points in nearly the same direction, we were able to use knowledge-based fragment growth to quickly elaborate this series to a number of analogs (Table 2), many with suitable properties to study them in vivo.

While the indazole SAR was useful in prediction of enzymatic affinity, prediction of the cellular potency was not as straight forward. Note that while compounds 10-12 are structurally quite similar, and the pIC50's are in the same range, the pEC50's vary somewhat depending on the placement of a methyl group. 
This effect became more pronounced in cases such as compounds $\mathbf{1 3}$ and $\mathbf{1 4}$ which performed identically in the enzyme assay but diverged in the cell assay by 100 fold.

It is of note that as the inhibitors grew modestly, the CYP1A2 inhibition exhibited by the smaller compounds from Table 1 was alleviated. The smallest compound (15) displays potent inhibition of CYP1A2, while a slight larger compound such as $\mathbf{1 0}$ showed moderate inhibition while the rest showed none. This can be explained by the relatively small binding pocket of CYP1 $\mathrm{A} 2^{5}$ which cannot accommodate larger ligands.

Table 2. Selected N-aryl pyrazolo[4,3-b]indoles

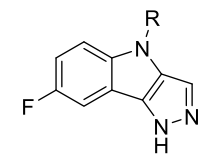

\begin{tabular}{|c|c|c|c|c|c|c|c|}
\hline Compd. & Structure (R) & $\operatorname{clog} \mathrm{D}$ & $\begin{array}{c}\text { MetAP2 } \\
\text { pIC }_{50}\end{array}$ & LE & $\overline{\mathrm{pEC}_{50}}$ & $\begin{array}{c}\text { CYP1A2 } \\
\text { pIC }_{50}\end{array}$ & $\begin{array}{c}\text { Human } \\
\mathrm{E}_{\mathrm{h}}{ }^{*}\end{array}$ \\
\hline 10 & & 1.7 & 8.3 & 0.49 & 7.9 & 5.5 & $<0.28$ \\
\hline 11 & & 2.2 & 8.0 & 0.47 & 6.6 & $<4.3$ & $<0.28$ \\
\hline 12 & & 1.8 & 8.0 & 0.46 & 7.6 & 4.7 & 0.33 \\
\hline 13 & & 0.9 & 8.2 & 0.45 & 5.7 & 5.1 & \\
\hline 14 & & 1.4 & 8.2 & 0.43 & 7.7 & 5.1 & $<0.28$ \\
\hline 15 & & 1.1 & 8.1 & 0.52 & 7.5 & 6.8 & 0.76 \\
\hline
\end{tabular}

* Stability in human liver microsomes at $1 \mu \mathrm{M}$. Data reported as extraction ratios $\left(\mathrm{E}_{\mathrm{h}}\right)$.

A co-crystal structure ${ }^{6}$ of compound $\mathbf{1 0}$ confirmed our presumption of its binding and interactions with the protein (Fig. 4). The pyrazole warhead N2 interacts directly with one metal ion (M1) while the N1-H makes a water mediated interaction with the other metal ion (M2). The phenyl ring of the core is well situated to make an edge/face interaction with Tyr-444. The 7-fluoro substituent protrudes into the lipophilic pocket occupied by the thioether of the endogenous ligands, making the ligand very shape complementary with the target. The aza-benzimidazole substituent at the N-4 position points into a hydrophobic space between Tyr-444 and His-339. 


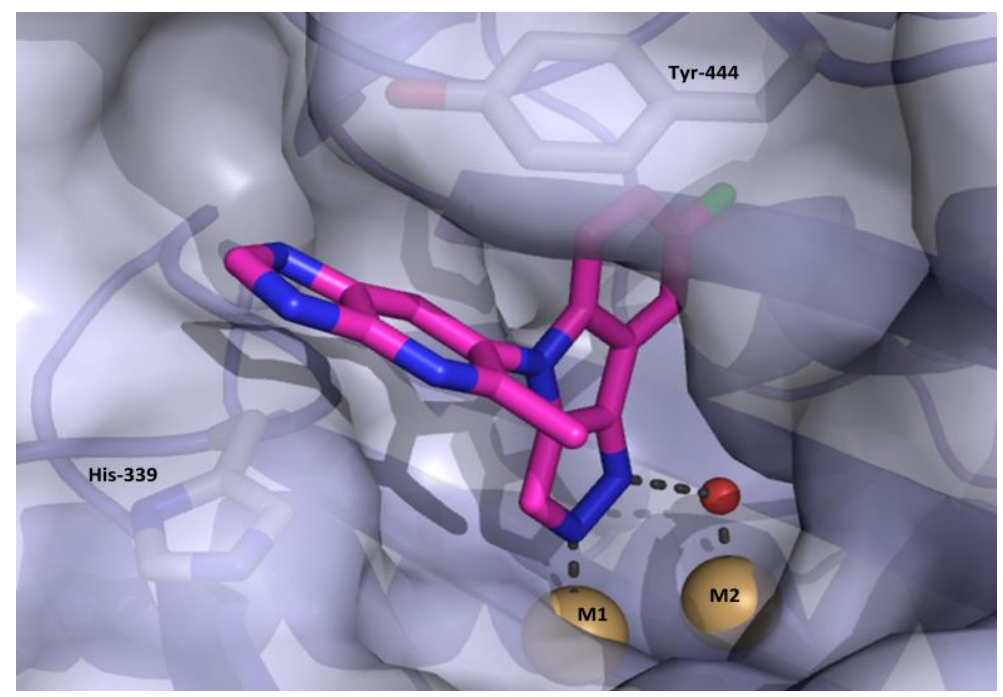

Fig. 4 Crystal structure of $\mathbf{1 0}$ with MetAP2 (pdb code: 5JFR)

We elected to investigate the pharmacokinetic profile of compound $\mathbf{1 0}$ due to its good enzyme and cellular potency, and good stability in microsomes (rat $E_{h}<0.19$, human $E_{h}<0.28$ ) and hepatocytes (human $E_{h}$ 0.27). When dosed orally at at $2.9 \mathrm{mg} / \mathrm{kg}$ in Sprague Dawley rats, this compound's pharmacokinetic profile showed acceptable exposure $(\mathrm{AUC}=2237 \mathrm{ng} * \mathrm{~h} / \mathrm{ml})$, an oral half-life of $6 \mathrm{~h}$, clearance of 17.2 $\mathrm{mL} / \mathrm{min} / \mathrm{kg}$, volume of distribution of $1597 \mathrm{~mL} / \mathrm{kg}$, and an oral bioavailability of $70 \%$.

With acceptable pharmacokinetics, compound $\mathbf{1 0}$ was further evaluated in a DIO-mouse model for obesity. Dose-dependent body weight loss (BWL) was observed when DIO mice were administered 10 orally for 7 days at $30 \mathrm{mg} / \mathrm{kg}$ qd (Fig. 5). At this dose, we observed a $4 \%$ reduction in body weight relative to the vehicle. Some BWL was seen in the vehicle in this study. This is a typical response of DIO mice to the stress of oral gavage. For some compounds we had the time acclimate the animals to the stress of oral gavage for a few weeks, until the DIO weights were not changing due to the stress of oral gavage before running the study. In these studies we saw similar results in the BWL with respect to the vehicle group. Importantly, one can see very little difference in the food intake between dosed and vehicle cohorts, one indication that the compounds are not toxic at this dose. Additionally, we monitored the activity levels of the animals and there was no difference between the vehicle and dosed groups.
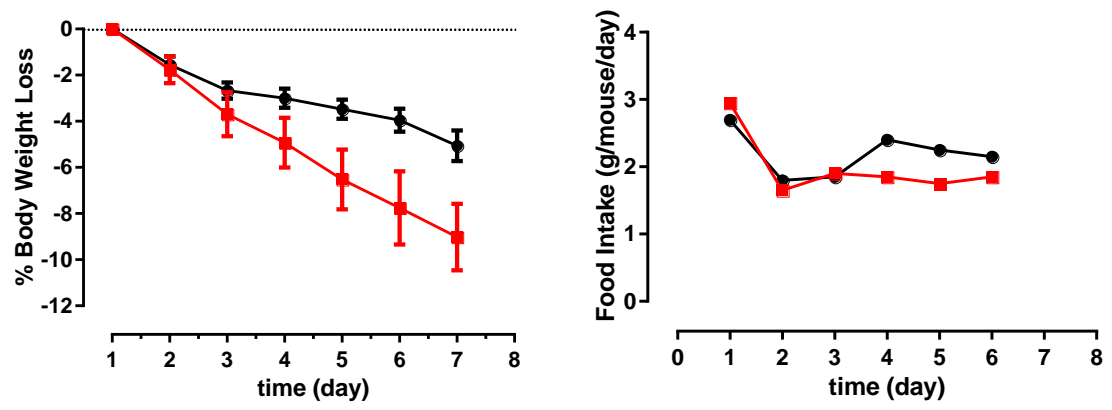

- Vehicle $(0.5 \% \mathrm{MC})$

$-1030 \mathrm{mg} / \mathrm{kg}$ gd $x 7$

Fig. 5 Body weight loss resulting from oral dosing of $\mathbf{1 0}$

Compound 10 was evaluated in a Ricerca Comprehensive Pharmacological Profile panel including 100 biological targets (GPCRs, ion channels, transporters and enzymes) and a panel of proteases and was found to have a no significant off target activity at $10 \mu \mathrm{M}(<50 \%$ inhibition). 
In summary, using a FBDD approach, a 6-substituted indazole core was identified as a potent inhibitor of MetAP2. From this fragment hit, SBDD was used to design a pyrazolo[4,3-b]indole core which was elaborated using an accelerated knowledge-based approach to deliver small, potent, selective, and reversible MetAP2 inhibitors. Compounds from this series have been developed with good pharmacokinetic properties in pre-clinical species and have shown efficacy in vivo.

\section{Acknowledgements}

We acknowledge the ADME group for generating the microsomal and solubility data, Jennifer Matuszkiewicz for helping to generate the cellular data, and Lisa Rahbaek for generating the in vivo PK data. The staff of the Berkeley Center for Structural Biology is gratefully acknowledged for support of beam line 5.0.3 at the Advanced Light Source. The Advanced Light Source is supported by the Director, Office of Science, Office of Basic Energy Sciences, of the U.S. Department of Energy under Contract No. DE-AC02-05CH11231.

\section{References and Notes:}

1. Cheruvallath et al, Bioorganic and Medicinal Chemistry Letters, preceding article

2. Marc Pudlo, Dorottya Csanyi, Fabien Moreau, Gyorgy Hajos, Zsuzsanna Riedl and Janos Sapi, Tetrahedron 2007, 63, 10320-10329

3. Patent applications describing our MetAP2 inhibitors have been published. Full experimental procedures for synthesis of all analogs in this paper along with enzymatic and cellular assay conditions are contained within the patent application. The screen was run both in the presence of Co and Mn. The data reported in this communication is from the Mn screen: WO2014039831(A1)

4. Molecular Operating Environment (MOE) software, MOE, Chemical Computing Group Inc.

5. Stefaan Sansen, Jason K. Yano, Rosamund L. Reynald, Guillaume A. Schoch, Keith J. Griffin, C. David Stout, and Eric F. Johnson, J.Biol.Chem. 2007 282: 14348-14355

6. The MetAp2 catalytic domain (residues 110-478) was cloned into the pFastBacHTb vector, and recombinant baculovirus was generated and expressed in fusion with an $\mathrm{N}$-terminal 6x poly-histidine tag and TEV cleavage site. Large scale production of recombinant protein was carried out in Spodoptera frugiperda Sf9 cells. The cell pellet was suspended into lysis buffer consisting of $25 \mathrm{mM}$ HEPES (pH 8.0), $1 \mathrm{M} \mathrm{NaCl}, 20 \mathrm{mM}$ imidazole, 3 Roche cOmplete Protease Inhibitor Tablets. The lysate was centrifuged, and clarified supernatant was batch bound with $10 \mathrm{ml}$ of Probond Ni resin (Invitrogen). The protein was then eluted with buffer containing $25 \mathrm{mM}$ HEPES ( $\mathrm{pH} 7.6$ ), $150 \mathrm{mM} \mathrm{NaCl}$, and $250 \mathrm{mM}$ imidazole. After tag cleavage, the protein was further purified by size-exclusion chromatography utilizing a Superdex 200 column equilibrated in $25 \mathrm{mM}$ HEPES (pH 7.6), $150 \mathrm{mM} \mathrm{NaCl}$, and 5\% Glycerol. For compound cocrystallization experiment, an aliquot of the frozen protein was incubated with the compound (final concentration $1 \mathrm{mM}$ ) and $\mathrm{MnCl} 2$ (final $0.3 \mathrm{mM}$ ) in $4^{\circ} \mathrm{C}$ overnight and then further concentrated the protein to $18 \mathrm{mg} / \mathrm{ml}$.

Crystals suitable for data collection are grown by vapor diffusion in sitting drops at $4{ }^{\circ} \mathrm{C}$ with $50 \mathrm{~nL}$ of protein solution and $50 \mathrm{~nL}$ of reservoir solution containing $38-42 \%$ MPD and 0.1M HEPES pH 6.8 -7.2.

Single crystals were immersed in mother liquor solution containing $22 \%$ ethylene glycol for cryoprotection and flash frozen. X-ray diffraction data to a resolution of $1.7 \AA$ was collected on an ADSC Q315R detector from single cryogenically protected crystals. Crystals of Metap2 complex belong to the orthorhombic space group $\mathrm{C} 222_{1}$ with unit cell dimensions $\mathrm{a}=89.64 \AA, \mathrm{b}=100.82 \AA, \mathrm{c}=99.44 \AA$, and contain one enzyme molecule in the asymmetric unit. Data was reduced using the HKL2000 software package. The structure was determined by molecular replacement with either MOLREP or PHASER of the CCP4 program suite and refined with the program REFMAC. Several cycles of model building with XtalView or COOT and refinement were performed for improving the quality of the model. The coordinates and structure factors have been deposited in Protein Data Bank with accession code 5JFR. 
<smiles>FC(F)(F)c1ccc2cn[nH]c2c1</smiles>

4

pIC50 7.3

LE $\quad 0.77$

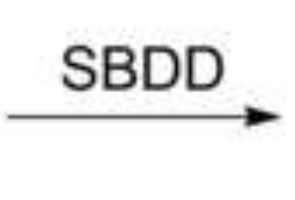

5

pIC50 6.0

LE $\quad 0.68$
Knowledge-based

Fragment growth

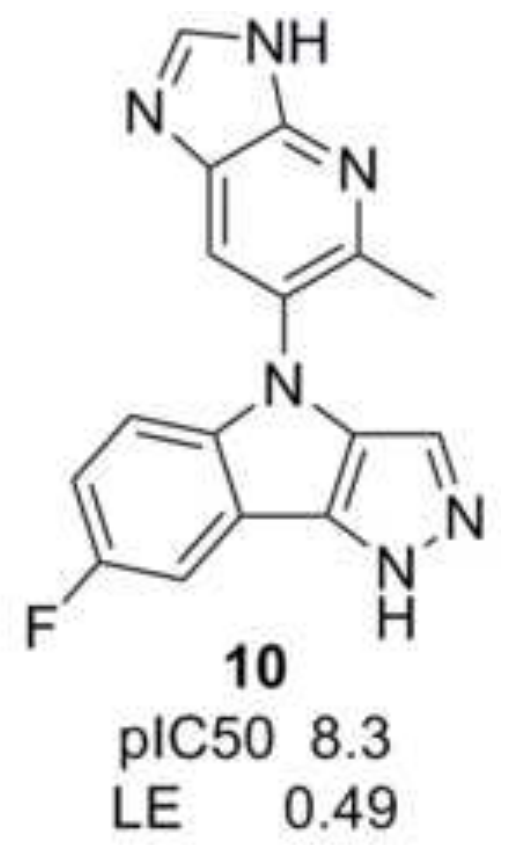

